\title{
Best-worst multi-criteria decision-making method: A robust approach
}

\author{
Seyed Jafar Sadjadi ${ }^{a^{*}}$ and Mahdi Karimi ${ }^{b}$
}

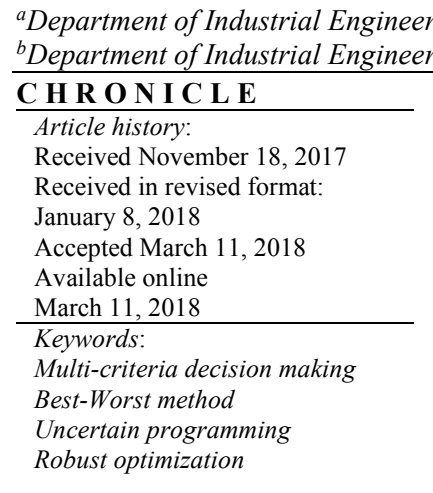

\section{Introduction}

Decision making plays an essential role in today's business activities. When a decision is made, it is necessary to consider different criteria, which may often be in conflict with each other. During the past few years, there was tremendous efforts to introduce multi criteria decision making (MCDM) techniques to find efficient ranking decisions. Analytical hierarchy process (AHP) is believed to be one of the pioneer methods introduced by Saaty (1980). The method uses a pair-wise comparison and using a simple but sophisticated method provides appropriate weights for different criteria. AHP normally uses $n(n-1) / 2$ comparisons to find the appropriate weights of the alternatives. When there are relatively large numbers of criteria, the comparisons will be increased and the process becomes time consuming and frustrating. One consequence of comparing too many criteria is the inconsistency, which appears in the final results. Rezaei (2015) proposed a method, which uses only the best and the worst criteria for pair-wise comparison. The method needs only $2 n-3$ comparisons, which makes it easier to use. In fact, Best-Worst method (BWM) is an MCDM method, where the decision maker chooses the best and the worst criteria and two pairwise comparison vectors for the best and the worst criteria are provided by the decision maker so this method requires fewer comparisons. Rezaei (2016) proposed a linear programming technique to determine the final weights of various criteria and Rezaei et al. (2016) implemented the BWM for different case studies.

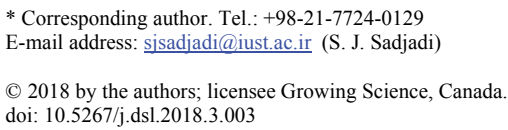


During the past two years, there have been a significant number of studies associated with BWM implementations. Rezaei et al. (2015) used best-worst method to make a connection between supplier development and supplier segmentation. You et al. (2016) studied BWM and ELECTRE method (Roy, 1968) in a group decision-making problem. Yang et al. (2016) implemented BWM for assessment of abroad talents in a real-world case study in China. Salimi and Rezaei (2016) used the BWM to measure the efficiency of Ph.D. projects. Sustainability assessment of technologies in an Eco-city based on bestworst method was studied by Ren et al. (2016). Mou et al. (2016) presented a fuzzy multiplicative BWM for a group decision-making problem. Gupta and Barua (2016) determined enablers of technological innovation for Indian micro, small and medium enterprises based on BWM method. Gupta and Barua (2017) used BWM and The Technique for Order of Preference by Similarity to Ideal Solution (TOPSIS) (Hwang \& Yoon, 1981) for green supplier selection among SMEs. The comparison of the type of energy source of electric vehicles using BWM is the main idea of the work of van de Kaa et al. (2017). Guo and Haoran (2017) developed BWM in a fuzzy environment and used it in several applications. Hafezalkotob and Hafezalkotob (2017) investigated both individual and group decisionmaking using a fuzzy BWM. The BWM was used to study the key success factors in technological innovation development (Ghaffari et al., 2017). Ahmad et al. (2017) used BWM to determine the effects of external forces on a gas supply chain. Shojaei et al. (2017) and Gupta (2017) made a comparison between BWM and Vlse Kriterijumska Optimizacija I Kompromisno Resenje (VIKOR) (Opricovic \& Tzeng, 2007) method. Gupta et al. (2017) used BWM to develop a strategy to dominate barriers to energy efficiency in buildings. BWM has been extensively used by several researchers (Salimi, 2017; Mohaghar, et al., 2017; van de Kaa, et al., 2017). Abadi et al. (2018) applied BWM in an appraisal of medical tourism development strategy in a real-world case study. Salimi and Rezaei (2018) used BWM to evaluate firms' research and development. Askarifar et al. (2018) used both BWM and TOPSIS to present an investment strategy in Iran's seashores.

This paper presents an application of robust optimization technique to BWM. The resulted robust approach is formulated as a linear programming where it can be solved using any commercial software package. The proposed model has been implemented on several instances which exist in the literature. This paper is organized as follows. We first present the problem statement of the BWM in section 2. Next, we explain robust optimization and offer robust BWM in section 3. Then, we use several instances from the literature and compare the results of both crisp and robust in section 4. Finally, conclusion remarks are given to summarize the contribution of this paper.

\section{Best Worst method}

An MCDM problem is made of some alternatives $\left(a_{1}, a_{2}, a_{3}, \ldots, a_{m}\right)$, multiple criteria $\left(c_{1}, c_{2}, c_{3}, \ldots, c_{n}\right)$ and each alternative has a score with respect to each criterion $\left(p_{11}, p_{12}, p_{13}, \ldots, p_{m n}\right)$. Therefore, an MCDM problem can be shown as the following matrix:

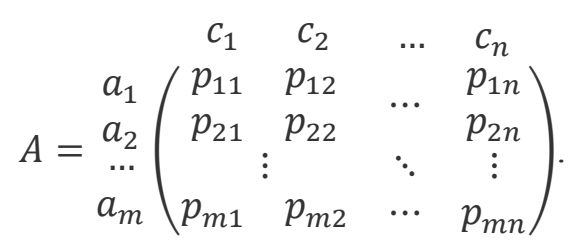

The main purpose of an MCDM problem is to find the best alternative with the best overall score $\left(V_{i}\right)$. There are many different methods to calculate the overall score for each alternative and the simplest way is to use an additive weighted value function as the following formula (Keeney \& Raiffa, 1993),

$$
V_{i}=\sum_{j=1}^{n} w_{j} p_{i j}
$$


We use best-worst method (BWM) (Rezaei, 2015) to determine the weight of each criterion $\left(w_{j}\right)$. Since we have the score of each alternative with respect to each criterion $\left(p_{i j}\right)$, the overall score can easily be obtained.

Based on BWM we need to track the following steps to calculate the vector $w=\left\{w_{1}, w_{2}, w_{3}, \ldots, w_{n}\right\}$ (Rezaei, 2015).

Step 1. Define a set of criteria

In this step the decision-maker should define a set of criteria $\left(\left\{c_{1}, c_{2}, c_{3}, \ldots, c_{n}\right\}\right)$ that is used to make a decision about alternatives.

Step 2. Define the best and the worst criterion

At this point, we ask the decision-maker to determine the best and the worst criterion regarded to their importance.

Step 3. Define the preferences of the best criterion over the other criteria

In this step the decision-maker determines a vector called Best-to-Other (BO) which is as below:

$$
A_{B}=\left(a_{B 1}, a_{B 2}, a_{B 3}, \ldots, a_{B n}\right)
$$

where $a_{B j}$ is the preference of the best criterion $B$ over the criterion $j$ and its value is an integer number in the range of 1 to 9 . Note that $a_{B B}=1$.

Step 4. Define the preferences of all criteria $(j)$ over the worst criterion $W$

In this step, the decision-maker determines a vector called Other-to-Worst $(\mathrm{OW})$ which is similar to the following vector:

$$
A_{W}=\left(a_{1 W}, a_{2 W}, a_{3 W}, \ldots, a_{n W}\right)^{T},
$$

where $a_{j W}$ is the preference of the criterion $j$ over the worst criterion $W$ and its value is an integer number in the range of 1 to 9 . Note that $a_{W W}=1$.

Step 5. Search for the optimal solution

In this step we must find the optimal weights of the criteria (vector $W$ ). To do this, we must find a solution, which minimizes the maximum gaps between obtained weights and the opinion of decisionmaker. Note that based on DM's opinion, $a_{j W}\left(a_{B j}\right)$ is the preference of the criterion $j$ over criterion $W$ (criterion $B$ over criterion $j)$, on other words, $a_{j W}=$ preference $j /$ preference $W\left(a_{B j}=\right.$ preference $B /$ preference $j$ ). In fact we determine the preference of each criterion when we find its weight, hence we can say $w_{j}=$ preference $j$. Therefore, we have preference $j /$ preference $W=$ $w_{j} / w_{w}$ (preference $B /$ preference $j=w_{B} / w_{j}$ ). The purpose of BWM is to find the vector of weights such that for each $\mathrm{j}$ absolute gaps $\left|w_{j} / w_{B}-a_{j w}\right|$ and $\left|w_{B} / w_{j}-a_{B j}\right|$ is minimized. To obtain this goal a mini-max formulation is presented, then a linear model is developed. (Rezaei, 2016)

We have the following model as the final linear model of BWM.

$\min \xi^{L}$

s.t.

$\left|w_{B}-a_{B j} w_{j}\right| \leq \xi^{L}$, for all $j$ 


$$
\begin{aligned}
& \left|w_{j}-a_{j W} w_{W}\right| \leq \xi^{L}, \text { for all } j \\
& \sum_{j=1}^{n} w_{j}=1 \\
& w_{j} \geq 0, \quad \text { for all } j
\end{aligned}
$$

The problem (5) has a unique solution and by solving it we will obtain the optimal weights $\left(w^{*}\right)$ and $\xi^{L *}$. We can consider $\xi^{L *}$ as an indicator of the consistency of the comparisons. The lower values of $\xi^{L *}$ represent higher consistency.

\section{A robust approach for Best-Worst Method}

In an MCDM problem we have some criteria (for example price, safety, and style, when we want to make a decision to buy a car), and in pairwise comparison methods, we need to ask decision-maker (DM) to compare these criteria. In previous methods, we ask him/her to assign a number to indicate the preferences of one criterion over another one (for example safety over the style). This is not realistic since it is hard to assign an exact value which shows the preference of two qualitative (or even quantitative) criteria over each other. So, this makes our data more unreliable. Applying uncertainty to pairwise comparisons is the main idea of this paper. It is more realistic to ask DM to assign an interval to compare two criteria instead of one crisp number. For example, using the interval $[7,9]$ instead of number 8 to compare safety and style is more rational and can be used easier by decision-maker which are often common people. One of the drawbacks of the previous method is the lack of attention to the uncertainty of parameters. On the other hand, even if the decision-maker assigns an exact value to the preference of one criterion over another one (for example 7), it is rational to add some uncertainty as the error of his/her opinion (for example $7 \pm 0.5$ ) to obtain a robust solution regarding to errors. In fact, we use robust optimization to make our decisions more reliable in an uncertain situation. In this section, we develop different robust approaches for BWM to handle these issues and make our decision more realistic.

We need to track the following steps to obtain the optimal weight of each criterion based on robust BWM. (Note that the first and second steps are same as the old BWM)

Step 1. Define a set of criteria

In this step the decision-maker should define a set of criteria $\left(\left\{c_{1}, c_{2}, c_{3}, \ldots, c_{n}\right\}\right)$ that is used to make a decision about alternatives.

Step 2. Define the best and the worst criterion

We ask the decision-maker to determine the best and the worst criterion regarded to their importance. Assume that we have only one best and one worst criterion.

Step 3. Determine the pairwise comparisons

Assigning the preferences values of each criterion over other criteria is same as the old method, but we use intervals instead of exact numbers.

We ask the decision maker to assign a continuous interval as the preference of the best criterion $B$ over all other criteria $j$. The result would be the Best-to-Other (BO) vector as following:

$$
A_{B}=\left(\tilde{a}_{B 1}, \tilde{a}_{B 2}, \tilde{a}_{B 3}, \ldots, \tilde{a}_{B n}\right),
$$


where $\tilde{a}_{B j}$ is the preference of the best criterion $B$ over the criterion $j$ and its value is a continuous interval between 0 and $9+e\left(e=\right.$ error, $\left.\tilde{a}_{B j}>0\right)$. We have $\tilde{a}_{B j}=\left[\tilde{a}_{B j}^{\prime}, \tilde{a}_{B j}^{\prime \prime}\right]$ or $\tilde{a}_{B j}=\dot{a}_{B j} \pm \hat{a}_{B j}, \dot{a}_{B j}$ is the center and $\hat{a}_{B j}$ is the width of interval, hence we have: $\dot{a}_{B j}=\left(\tilde{a}_{B j}^{\prime}+\tilde{a}_{B j}^{\prime \prime}\right) / 2$ and $\hat{a}_{B j}=$ $\left(\tilde{a}_{B j}^{\prime \prime}-\tilde{a}_{B j}^{\prime}\right) / 2$. In special cases, we can assume that $\dot{a}_{B j}$ is the exact value used in the old method and $\hat{a}_{B j}$ is the error of decision-maker's opinion.

The decision maker should assign a continuous interval to preference each criterion $j$ over the worst criterion $W$. The result would be the Other-to-Worst (OW) vector as following:

$$
A_{W}=\left(\tilde{a}_{1 W}, \tilde{a}_{2 W}, \tilde{a}_{3 W}, \ldots, \tilde{a}_{n W}\right)^{T}
$$

Similar to BO vector, $\tilde{a}_{l W}$ is the preference of the criterion $j$ over the worst criterion $W$ and its value is a continues interval between 0 and $9+e\left(e=\right.$ error, $\left.\tilde{a}_{j W}>0\right)$. So $\tilde{a}_{j W}=\left[\tilde{a}_{j W}^{\prime}, \tilde{a}^{\prime \prime}{ }_{j W}\right]$ or $\tilde{a}_{j W}=\dot{a}_{j W} \pm$ $\hat{a}_{j W}, \dot{a}_{j W}$ is the center and $\hat{a}_{j W}$ is the width of interval, so, $\dot{a}_{j W}=\left(\tilde{a}_{j W}^{\prime}+\tilde{a}^{\prime \prime}{ }_{j W}\right) / 2$ and $\hat{a}_{j W}=$ $\left(\tilde{a}_{j W}^{\prime \prime}-\tilde{a}_{j W}^{\prime}\right) / 2$. Similarly, we can assume that $\dot{a}_{j W}$ is the exact value used in the old method and $\hat{a}_{j W}$ is the error of decision-maker's opinion.

Note that $\tilde{a}_{B B}=\tilde{a}_{W W}=1$, in other words comparing a criterion over itself has no error and $\tilde{a}_{B B}, \tilde{a}_{W W}$ can be only an exact value (only 1 ), hence we have $\tilde{a}_{B B}=\tilde{a}_{W W}=1 \pm 0$. Note that we always have the same intervals for pairwise comparisons between the best and the worst criteria so we must have the same interval for $\tilde{a}_{B W}$ in two vectors.

In this step, we must be careful about determining the error in a way which the value of each comparison crosses the limits (interval $(0,9+\mathrm{e}])$. The value of the error can be greater than 1 , but the higher error shows lower trust on decision-maker's opinion. Note that the value of error for each pairwise comparison may differ with others, for example when we want to make a pairwise comparison between the cost of two cars, we can divide their values by each other but it is different when we compare the safety since the values are linguistic and we cannot obtain the value of pairwise comparison easily. In fact, when we compare the price of two cars the value of error is lower than comparing their safety. According to what was argued it is logical to assume different values of error for each comparison.

Step 4. Find the best solution

In this step we find the best set of weights to minimize the absolute differences between the obtained results and the opinion of the decision-maker. To achieve this purpose, we develop the model (5) with different robust approaches.

\section{a) The robust formulation of Soyester for the best-worst method}

One of the primary methods for robust formulation was introduced by Soyester (1973). According to Soyester's method we have following model as the robust formulation of the model (5).

$$
\begin{array}{lc}
\min \xi^{R} & \\
\text { s.t. } & \\
w_{B}-\dot{a}_{B j} w_{j}+\hat{a}_{B j} y_{b}-\xi^{R} \leq 0, & \forall j, b \\
\dot{a}_{B j} w_{j}-w_{B}+\hat{a}_{B j} y_{b^{\prime}}-\xi^{R} \leq 0, & \forall j, b^{\prime} \\
w_{j}-\dot{a}_{j W} w_{W}+\hat{a}_{j W} y_{w}-\xi^{R} \leq 0, & \forall j, w \\
\dot{a}_{j W} w_{W}-w_{j}+\hat{a}_{j W} y_{w^{\prime}}-\xi^{R} \leq 0, & \forall j, w^{\prime} \\
-y_{b} \leq w_{j} \leq y_{b}, & \forall b, j \\
-y_{b^{\prime}} \leq w_{j} \leq y_{b^{\prime}}, & \forall b^{\prime}, j \\
-y_{w} \leq w_{W} \leq y_{w}, & \forall w, j
\end{array}
$$




$$
\begin{array}{ll}
-y_{w^{\prime}} \leq w_{W} \leq y_{w^{\prime}}, & \forall w^{\prime}, j \\
0 \leq w_{j} \leq 1, & \forall j \\
\sum_{j=1}^{n} w_{j}=1 & \\
W, Y \geq 0 &
\end{array}
$$

This method admits full protection of uncertainty which means the proposed model sets the uncertain parameters at their limits (their worst states) and this makes this method inflexible. In fact, we do not have any control on the degree of uncertainty. On the other hand, applying full protection would increase the inconsistency (minimum consistency occurs in maximum protection). This method has good performance in our study, but there are other methods to obtain better results. (for more details see Soyester, 1973).

\section{b) The robust formulation of Bertsimas and Sim for the best-worst method}

Based on the method presented by Bertsimas and Sim (2004), we developed the following model for best-worst method:

$$
\begin{array}{lc}
\min \xi^{R} & \\
\text { s.t. } & \\
w_{B}-\dot{a}_{B j} w_{j}+z_{b} \Gamma_{b}+p_{b}-\xi^{R} \leq 0, & \forall j, b \\
\dot{a}_{B j} w_{j}-w_{B}+z_{b^{\prime}} \Gamma_{b^{\prime}}+p_{b^{\prime}}-\xi^{R} \leq 0, & \forall j, b^{\prime} \\
w_{j}-\dot{a}_{j W} w_{W}+z_{w} \Gamma_{w}+p_{w}-\xi^{R} \leq 0, & \forall j, w \\
\dot{a}_{j W} w_{W}-w_{j}+z_{w^{\prime}} \Gamma_{w^{\prime}}+p_{w^{\prime}}-\xi^{R} \leq 0, & \forall j, w^{\prime} \\
& \forall j, b \\
z_{b}+p_{b} \geq \hat{a}_{B j} y_{b}, & \forall j, b^{\prime} \\
z_{b^{\prime}}+p_{b^{\prime}} \geq \hat{a}_{B j} y_{b^{\prime}}, & \forall j, w \\
z_{w}+p_{w} \geq \hat{a}_{j W} y_{w}, & \forall j, w^{\prime} \\
z_{w^{\prime}}+p_{w^{\prime}} \geq \hat{a}_{j W} y_{w^{\prime}}, & \forall b, j \\
-y_{b} \leq w_{j} \leq y_{b}, & \forall b^{\prime}, j \\
-y_{b^{\prime}} \leq w_{j} \leq y_{b^{\prime}}, & \forall w, j \\
-y_{w} \leq w_{W} \leq y_{w}, & \forall w^{\prime}, j \\
-y_{w^{\prime}} \leq w_{W} \leq y_{w^{\prime}}, & \forall j \\
0 \leq w_{j} \leq 1, & \\
n & \\
\sum w_{j=1}=1 & \\
W, Y, Z, P \geq 0, &
\end{array}
$$

In this model, added variables $(Z, P, Y)$ are the robustness variables and the positive parameter $\Gamma_{i}$ denotes the budget of uncertainty for the $i$ th constraint. In the problem (9), each constraint has only one variable with an uncertain coefficient, hence $n=\left|J_{i}\right|=1 \forall i$, so if we let $\Gamma_{i}=1 \forall i$, we have Soyester's method. If we let $\Gamma_{i}=0 \forall i$, the problem is equivalent to the nominal problem (problem 
(5)). Note that by varying $\Gamma_{i} \in[0,1]$ the flexibility of adjusting the robustness versus the level of conservatism of the solution would be obtained. The probability that each constraint is violated (error in results) is $1-\varphi\left(\left(\Gamma_{i}-1\right) / \sqrt{n}\right)$ so for $\Gamma_{i} \in[0,1]$ there are such a high probability of errors which makes our results unreliable. For full protection the value of error is $50 \%$. This is a high value of risks which makes our results unreasonable, so this method has not good performance in our study and we must use another method to obtain more reliable results. (for the full details of robustness method, see (Bertsimas \& Sim, 2004).

\section{c) The robust formulation of Ben-Tal and Nemirovski for the best-worst method}

One of the robust formulations was introduced by Ben-Tal and Nemirovski (Ben-Tal \& Nemirovski, 2000). This method functions well and we have the ability to control the degree of uncertainty however did not gain much attention since it is nonlinear. We implemented this method to the model (5). Because there is only one variable with uncertain coefficient in each constraint of our model, we could rewrite the nonlinear model into a linear one. Finally, we developed a linear model based on of Ben-Tal approach for BWM as following:

$$
\min \xi^{R}
$$

s.t.

$$
\begin{aligned}
& w_{B}-\dot{a}_{B j} w_{j}+\hat{a}_{B j} y_{b}+\Omega_{b} \hat{a}_{B j} z_{b}-\xi^{R} \leq 0, \quad \forall j, b \\
& w_{B}-\dot{a}_{B j} w_{j}+\hat{a}_{B j} y_{b}-\Omega_{b} \hat{a}_{B j} z_{b}-\xi^{R} \leq 0, \quad \forall j, b \\
& \dot{a}_{B j} w_{j}-w_{B}+\hat{a}_{B j} y_{b^{\prime}}+\Omega_{b^{\prime}} \hat{a}_{B j} z_{b^{\prime}}-\xi^{R} \leq 0, \quad \forall j, b^{\prime} \\
& \dot{a}_{B j} w_{j}-w_{B}+\hat{a}_{B j} y_{b^{\prime}}-\Omega_{b^{\prime}} \hat{a}_{B j} z_{b^{\prime}}-\xi^{R} \leq 0, \quad \forall j, b^{\prime} \\
& w_{j}-\dot{a}_{j W} w_{W}+\hat{a}_{j W} y_{w}+\Omega_{w} \hat{a}_{j W} z_{w}-\xi^{R} \leq 0, \quad \forall j, w \\
& w_{j}-\dot{a}_{j W} w_{W}+\hat{a}_{j w} y_{w}-\Omega_{w} \hat{a}_{j W} z_{w}-\xi^{R} \leq 0, \quad \forall j, w \\
& \dot{a}_{j W} w_{W}-w_{j}+\hat{a}_{j W} y_{w^{\prime}}+\Omega_{w^{\prime}} \hat{a}_{j W^{\prime} z_{w \prime}}-\xi^{R} \leq 0, \quad \forall j, w^{\prime} \\
& \dot{a}_{j W} w_{W}-w_{j}+\hat{a}_{j W} y_{w^{\prime}}-\Omega_{w^{\prime}} \hat{a}_{j W^{\prime} z_{w \prime}}-\xi^{R} \leq 0, \quad \forall j, w^{\prime} \\
& -y_{b} \leq w_{j}-z_{b} \leq y_{b}, \\
& -y_{b^{\prime}} \leq w_{j}-z_{b^{\prime}} \leq y_{b^{\prime}} \text {, } \\
& \forall b, j \\
& \forall b^{\prime}, j \\
& -y_{w} \leq w_{W}-z_{w} \leq y_{w}, \\
& -y_{w^{\prime}} \leq w_{W}-z_{w^{\prime}} \leq y_{w^{\prime}} \text {, } \\
& \forall w, j \\
& \forall w^{\prime}, j \\
& 0 \leq w_{j} \leq 1, \\
& \forall j \\
& \sum_{j=1}^{n} w_{j}=1 \\
& W, Y \geq 0,
\end{aligned}
$$

In the model $(10)$ added variables $(Z, Y)$ are robustness variables and $\Omega$ is a positive parameter which shows the probability of crossing the limits (error in results). Assume that in the $i$ th constraint, $\hat{a}_{i j} \leq$ $e_{i j}\left|a_{i j}\right|$, so $e_{i j}$ is the degree of uncertainty for $a_{i j}$ and we can control our results by varying this parameter. In this model if we let $e=0$, since we want to minimize $\xi^{R}$, we have $Y=0$ and $W=Z$ in the optimum solution. In fact, for $e=0\left(\hat{a}_{i j}=0\right)$ the problem (10) is equivalent to the nominal 
problem. Note that $\Omega$ functions like $\Gamma$ in the previous model but as we argued, there is a high level of error in results of the Bertsimas's method. In this method, The probability that the constraint $i$ is violated (error in results) is lower than $\kappa=\exp \left(-\Omega_{i}{ }^{2} / 2\right)$, so we can control the level of errors in our results using $\Omega$. If we require high reliability we must assign large values to $\Omega$, for example if we want to $\kappa$ be at most $10^{-6}$ we must let $\Omega=5.24$ (this is a high reliability and the probability of violation of each constraint is $\left.10^{-6}\right)$. Also, we can vary $\Omega$ in the interval $(0,+\infty]$ and there is no limitation using this parameter to obtain more reliable results. So the model (10) functions better than previous methods and since it is linear, we can use any common solver to solve it. According to what we said, all of three developed models can be used in uncertain MCDM problems but in this study we use the model (10) because it has better performance than others.

Solving the problems (8), (9) and (10), the optimal weights $\left\{w_{1}^{*}, w_{2}^{*}, w_{3}^{*}, \ldots, w_{n}^{*}\right\}$ and $\xi^{R *}$ for the robust approach are obtained. Similar to the nominal model we can consider $\xi^{R *}$ as an indicator of the consistency of the comparisons. The lower values of $\xi^{R *}$ represent higher consistency.

In this section we developed three different robust approaches for uncertain MCDM problems, next, experimental results are presented.

\section{Experimental results}

In this section, we want to investigate the effect of applying uncertainty on the examples and study how uncertainty will change our decision-making. In fact, we want to study how to make our decisions robust, against errors.

\subsection{The effect of uncertainty (or errors) on the rank of criteria}

Example 1.

We want to study the effect of applying some errors to each parameter of the example.2 in the work of Rezaei (2016). The OW and BO vectors are shown in Table 1.

\section{Table 1}

Best-to-others $(\mathrm{BO})$ and others-to-worst $(\mathrm{OW})$ pairwise comparison vectors for Example 1

\begin{tabular}{|c|c|c|c|c|c|}
\hline $\mathrm{BO}$ & Quality & Price & Comfort & Safety & Style \\
\hline Best criterion: price & $2 \pm 0.25 *$ & 1 & $4 \pm 0.5$ & $3 \pm 0.3$ & $8 \pm 0.65$ \\
\hline OW & & & & & Worst criterion: style \\
\hline Quality & & & & & $4 \pm 0.25$ \\
\hline Price & & & & & $8 \pm 0.45$ \\
\hline Comfort & & & & & $2 \pm 0.15$ \\
\hline Safety & & & & & $3 \pm 0.25$ \\
\hline Style & & & & & 1 \\
\hline
\end{tabular}

We want to check the validation of our proposed model, so this example was solved with all three developed models to check their validation.

Table 2

Numerical results of example 1

\begin{tabular}{lcccccc}
\hline & $w_{1}^{*}$ & $w_{2}^{*}$ & $w_{3}^{*}$ & $w_{4}^{*}$ & $w_{5}^{*}$ & $\xi^{R *}$ \\
\hline Nominal Problem & 0.2295 & 0.4480 & 0.1147 & 0.1530 & 0.0546 & 0.0109 \\
Soyester(error=0) & 0.2295 & 0.4480 & 0.1147 & 0.1530 & 0.0546 & 0.0109 \\
Bertsimas $(\Gamma=0)$ & 0.2295 & 0.4480 & 0.1147 & 0.1530 & 0.0546 & 0.0109 \\
Ben-Tal $(e=0)$ & 0.2295 & 0.4480 & 0.1147 & 0.1530 & 0.0546 & 0.0109 \\
Soyester & 0.2251 & 0.4502 & 0.1125 & 0.1534 & 0.0585 & 0.0562 \\
Bertsimas $(\Gamma=1)$ & 0.2251 & 0.4502 & 0.1125 & 0.1534 & 0.0585 & 0.0562 \\
Ben-Tal $(e \neq 0, \Omega=5.24)$ & 0.2251 & 0.4502 & 0.1125 & 0.1534 & 0.0585 & 0.0562 \\
\hline
\end{tabular}


According to Table 2 for zero protection of uncertainty the results are same as the nominal problem in all of three methods. Although, these results show that in full protection, the results obtained from each method are congruous and the proposed models are valid.

If the protection increases some values will decrease $\left(w_{1}^{*}, w_{3}^{*}\right)$ and some other will increase $\left(w_{2}^{*}, w_{4}^{*}, w_{5}^{*}\right)$. Applying uncertainty will reduce the consistency of the comparisons but even in full protection the inconsistency indicator is not very high, on other words uncertainty does not cause inconsistency in model as much as could make the results unreliable. Note that in this example the ranking of the criteria did not change. When we use Eq. (2) to find the overall score of alternatives, we use the values, not the ranks. Since the values are changed it is possible that the overall scores ranking change. So as a result, adding same error to each pairwise comparison may cause a change in our decisions.

We use Ben-Tal approach in next examples.

\section{Example 2.}

For the second example, we use the same data as in the example.1 in the work of Rezaei (2016).

Here we add a random error (e) to each pairwise comparison of the main example. We assume that the opinion of the decision-maker has an error and we want to make our decision robust against it. Table 3 shows the BO and OW vectors when we decide to choose a car:

\section{Table 3}

Best-to-others $(\mathrm{BO})$ and others-to-worst $(\mathrm{OW})$ pairwise comparison vectors for Example 2

\begin{tabular}{|c|c|c|c|c|c|}
\hline $\mathrm{BO}$ & Quality & Price & Comfort & Safety & Style \\
\hline Best criterion: price & $2 \pm 5 \% *$ & 1 & $4 \pm 2 \%$ & $2 \pm 3 \%$ & $8 \pm 5 \%$ \\
\hline OW & & & & & Worst criterion: style \\
\hline Quality & & & & & $4 \pm 2 \%$ \\
\hline Price & & & & & $8 \pm 5 \%$ \\
\hline Comfort & & & & & $2 \pm 1 \%$ \\
\hline Safety & & & & & $4 \pm 2 \%$ \\
\hline Style & & & & & 1 \\
\hline
\end{tabular}

*We added a value to the main parameters as error and we can show this equation as an interval [1.9,2.1].

The problem (10) was solved with $\Omega=5.24$, the results are shown in table 4 .

Table 4

Numerical results of example 2

\begin{tabular}{|c|c|c|c|c|c|c|}
\hline$e$ & $w_{1}^{*}$ & $w_{2}^{*}$ & $w_{3}^{*}$ & $w_{4}^{*}$ & $w_{5}^{*}$ & $\xi^{R *}$ \\
\hline 0 & 0.2105 & 0.4210 & 0.1052 & 0.2105 & 0.0526 & 0 \\
\hline$\neq 0$ & 0.2090 & 0.4180 & 0.1075 & 0.2130 & 0.0522 & 0.0209 \\
\hline
\end{tabular}

The ranking of the criteria in this example is shown in table 5 and figure 1.

\section{Table 5}

The ranking of each criterion in example 2

\begin{tabular}{llllll}
\hline & Quality & Price & Comfort & Safety & Style \\
\hline Nominal problem & 2 & 1 & 4 & 2 & 5 \\
Uncertain problem & 3 & 1 & 4 & 2 & 5 \\
\hline
\end{tabular}

According to the results, as we expect for $e=0$ we obtain the same results as the original example, hence for $e=0$ the problem (10) is equivalent to the nominal one. The results show that even low uncertainty would change the ranking of the criteria. According to Table 4, applying uncertainty reduces the consistency of comparisons, but for $e \neq 0$ we have a low inconsistency rate (0.0209). High reliability $\left(\Omega=5.24, \kappa=10^{-6}\right)$ and consistency $(\cong 98 \%)$ show that applying uncertainty to the model would not reduce the rationality of the results. 


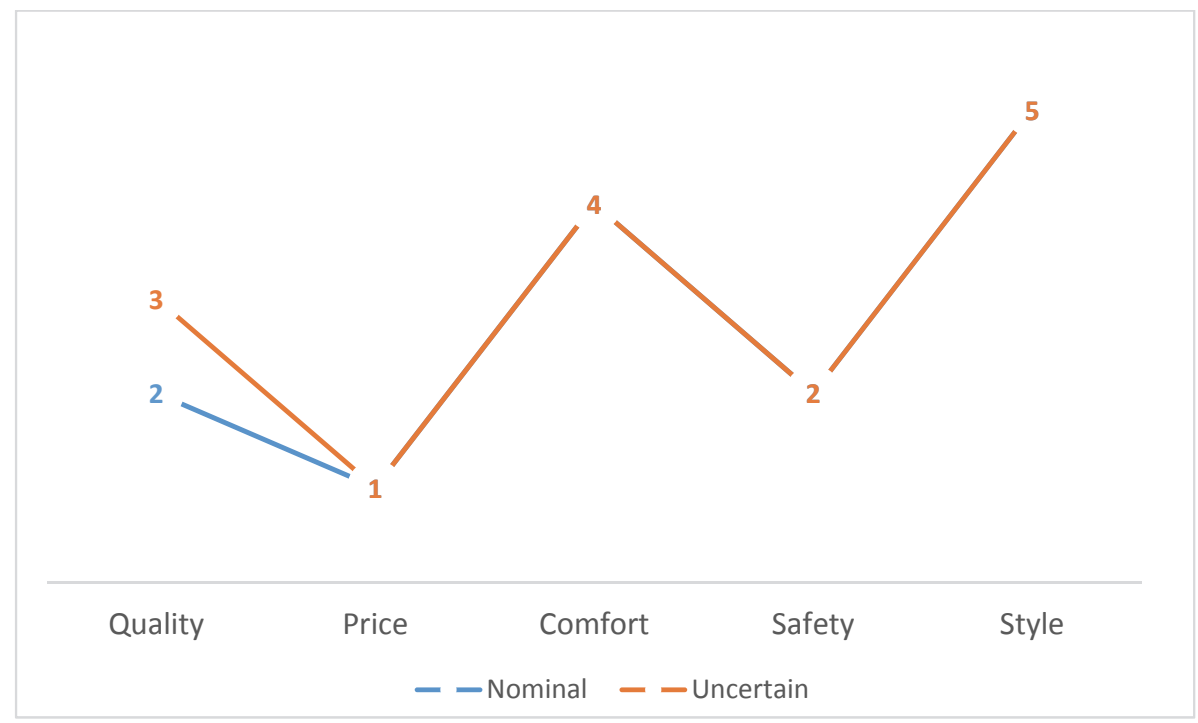

Fig 1. Compare of ranking of criteria in example 2

\section{Example 3.}

In this example, we want to investigate the effect of applying uncertainty for a real-world case study presented by Ghaffari et al. (2017).

We use the same data but with some error and investigate the effect of this error on the ranking of each sub-criterion. In this example, we consider the same value as the error for sub-criteria comparisons.

We assume that the main criteria comparisons error is, $e=0.55$ (Tables 3 and 7 in the main study).

The error of Actors and Network sub-criteria comparisons is, $e=0.3$ (Tables 4 and 8 in the main study).

For Institution sub-criteria vectors, we assume that error is, $e=0.45$ (Tables 5 and 9 in the main study).

The error of Science and technology sub-criteria vectors is, $e=0.5$ (Tables 6 and 10 in the main study).

By solving the problem (10) with high reliability $(\Omega=5.24)$ we obtain the following results.

\section{Table 6}

Obtained results when we let $e=0$ in example 3

\begin{tabular}{|c|c|c|c|c|c|}
\hline Criteria & Weight & Sub-Criteria & Local Weight & Global Weight & Rank \\
\hline \multirow{9}{*}{ K1 } & \multirow{9}{*}{0.526} & K11 & 0.048 & 0.025 & 13 \\
\hline & & K12 & 0.316 & 0.166 & 1 \\
\hline & & K13 & 0.079 & 0.041 & 9 \\
\hline & & K14 & 0.136 & 0.072 & 5 \\
\hline & & K15 & 0.100 & 0.053 & 7 \\
\hline & & K16 & 0.056 & 0.029 & 12 \\
\hline & & K17 & 0.173 & 0.091 & 3 \\
\hline & & K18 & 0.028 & 0.015 & 18 \\
\hline & & K19 & 0.061 & 0.032 & 11 \\
\hline \multirow{7}{*}{ K2 } & \multirow{7}{*}{0.200} & K21 & 0.096 & 0.019 & 16 \\
\hline & & K22 & 0.068 & 0.014 & 19 \\
\hline & & K23 & 0.034 & 0.007 & 21 \\
\hline & & K24 & 0.080 & 0.016 & 17 \\
\hline & & K25 & 0.211 & 0.042 & 8 \\
\hline & & K26 & 0.122 & 0.024 & 14 \\
\hline & & K27 & 0.387 & 0.077 & 4 \\
\hline \multirow{5}{*}{ K3 } & \multirow{5}{*}{0.273} & K31 & 0.129 & 0.035 & 10 \\
\hline & & K32 & 0.045 & 0.012 & 20 \\
\hline & & K33 & 0.091 & 0.023 & 15 \\
\hline & & K34 & 0.221 & 0.060 & 6 \\
\hline & & K35 & 0.513 & 0.140 & 2 \\
\hline
\end{tabular}


Since by ignoring the uncertainty in the problem (10) we must obtain the same results as the nominal problem, we let $e=0$, to investigate the verification of the proposed model. The results are shown in Table 6. The results are same as the main study, therefore the proposed model functions well. Note that there is a little gap in some values, it is the result of using different solvers and the gap is negligible. According to the comparison of the proposed method and the main studies, we find that the results obtained from the developed model are reliable. Then we apply uncertainty to example 2 , the results are shown in Table 7. A comparison between the nominal and the uncertain problem rankings is provided in Fig. 2.

Table 7

Obtained results when we let $e \neq 0$ in example 3

\begin{tabular}{|c|c|c|c|c|c|}
\hline Criteria & Weight & Sub-Criteria & Local Weight & Global Weight & Rank \\
\hline \multirow{9}{*}{ K1 } & \multirow{9}{*}{0.514} & K11 & 0.050 & 0.025 & 14 \\
\hline & & K12 & 0.327 & 0.168 & 1 \\
\hline & & K13 & 0.079 & 0.041 & 8 \\
\hline & & K14 & 0.131 & 0.067 & 5 \\
\hline & & K15 & 0.099 & 0.051 & 7 \\
\hline & & K16 & 0.057 & 0.029 & 12 \\
\hline & & K17 & 0.162 & 0.083 & 3 \\
\hline & & K18 & 0.029 & 0.015 & 18 \\
\hline & & K19 & 0.062 & 0.032 & 11 \\
\hline \multirow{7}{*}{$\mathrm{K} 2$} & \multirow{7}{*}{0.200} & K21 & 0.096 & 0.019 & 16 \\
\hline & & K22 & 0.070 & 0.014 & 19 \\
\hline & & K23 & 0.035 & 0.007 & 21 \\
\hline & & K24 & 0.081 & 0.016 & 17 \\
\hline & & K25 & 0.191 & 0.038 & 9 \\
\hline & & K26 & 0.119 & 0.024 & 15 \\
\hline & & $\mathrm{K} 27$ & 0.405 & 0.081 & 4 \\
\hline \multirow{5}{*}{ K3 } & \multirow{5}{*}{0.286} & K31 & 0.127 & 0.036 & 10 \\
\hline & & K32 & 0.045 & 0.013 & 20 \\
\hline & & K33 & 0.092 & 0.026 & 13 \\
\hline & & K34 & 0.204 & 0.058 & 6 \\
\hline & & K35 & 0.530 & 0.152 & 2 \\
\hline
\end{tabular}

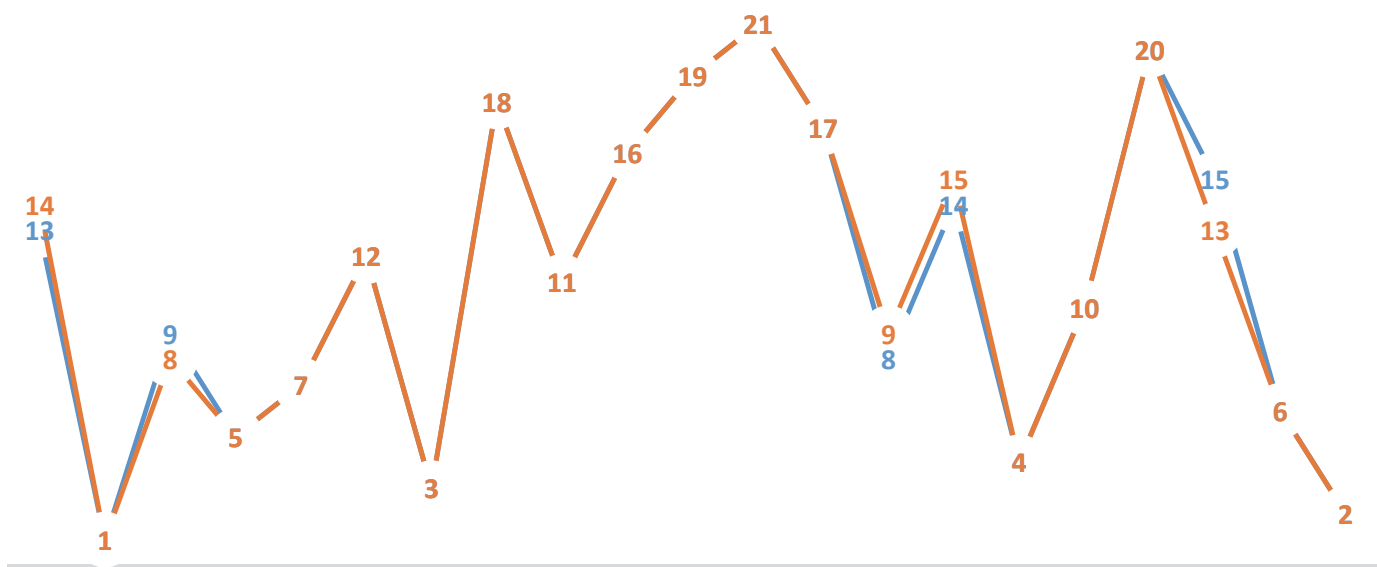

K11 K12 K13 K14 K15 K16 K17 K18 K19 K21 K22 K23 K24 K25 K26 K27 K31 K32 K33 K34 K35

$$
- \text { Nominal }- \text { Uncertain }
$$

Fig. 2. A compare between the rankings in the nominal and the uncertain problem 
According to Table 5 and Table 6 we find that applying uncertainty may change the ranking of the criteria. In this example, each sub-criterion yields the same ranking as the nominal problem but we investigate the ranking globally and at last, five criteria were ranked differently in the uncertain problem. Also, we see that the inconsistency indicator of the comparisons in the uncertain problem is not much greater than the nominal problem; hence applying uncertainty will not cause much inconsistency and would not reduce the rationality of the results.

As we see in these examples adding error (even very low) to data can change the ranking of the criteria and it may affect our decision, hence it is necessary to consider uncertainty in MCDM problems.

\subsection{The effect of uncertainty on the ranking of alternatives}

In this section, we study the effect of applying errors to the decision-maker's opinion to investigate changing in the ranking of the alternatives.

Example 4.

In this example a real-world problem for supplier selection is presented, we use the same data as used by Rezaei et al. (2016) to show the effect of uncertainty on the ranking of the alternatives. Instead of six respondents in the main study, we assume that there is only one respondent (respondent no. 1 in the main study) and there is only one type of raw material (type $C$ in the main study). We study following data set in this example. Pairwise comparisons are shown in Table 8. Table 9 shows the normalized score of each alternative with respect to the criteria.

Table 8

$\mathrm{BO}$ and $\mathrm{OW}$ vectors for example 4

\begin{tabular}{|c|c|c|c|c|c|c|c|c|}
\hline $\mathrm{BO}$ & $c_{1}$ & $c_{2}$ & $c_{3}$ & $c_{4}$ & $c_{5}$ & $c_{6}$ & $c_{7}$ & $c_{8}$ \\
\hline Best criterion: $c_{4}$ & 3 & 4 & 6 & 1 & 5 & 2 & 9 & 7 \\
\hline $\mathrm{OW}$ & & & & & & & & Worst criterion: $c_{7}$ \\
\hline$c_{1}$ & & & & & & & & 7 \\
\hline$c_{2}$ & & & & & & & & 6 \\
\hline$c_{3}$ & & & & & & & & 4 \\
\hline$c_{4}$ & & & & & & & & 9 \\
\hline$c_{5}$ & & & & & & & & 5 \\
\hline$c_{6}$ & & & & & & & & 8 \\
\hline$c_{7}$ & & & & & & & & 1 \\
\hline$c_{8}$ & & & & & & & & 3 \\
\hline
\end{tabular}

Table 9

Normalized suppliers score for raw material( $\mathrm{C}$ in the main study)

\begin{tabular}{lllllllll}
\hline Criterion & $c_{1}$ & $c_{2}$ & $c_{3}$ & $c_{4}$ & $c_{5}$ & $c_{6}$ & $c_{7}$ & $c_{8}$ \\
\hline Supplier A & 0.028 & 0.496 & 1.000 & 0.274 & 0.267 & 1.000 & 1.000 & 0.000 \\
Supplier B & 0.000 & 0.474 & 0.222 & 0.274 & 0.200 & 0.875 & 0.778 & 0.875 \\
Supplier C & 0.547 & 0.035 & 0.333 & 0.048 & 0.167 & 0.625 & 1.000 & 1.000 \\
Supplier D & 0.204 & 0.000 & 0.333 & 0.048 & 1.000 & 0.625 & 1.000 & 1.000 \\
Supplier E & 0.910 & 0.737 & 0.222 & 0.048 & 0.500 & 0.625 & 1.000 & 0.875 \\
Supplier F & 0.607 & 0.693 & 0.222 & 0.000 & 0.467 & 0.625 & 1.000 & 0.875 \\
Supplier G & 0.174 & 0.605 & 0.667 & 0.048 & 0.967 & 0.625 & 0.556 & 0.500 \\
\hline
\end{tabular}

The results of the nominal problem $(e=0)$ are shown in Tables 10 . 
Table 10

Weight and ranking of each criterion in the nominal problem of example 4.( $e=0$ in uncertain problem)

\begin{tabular}{lllllllll} 
Criterion & $c_{1}$ & $c_{2}$ & $c_{3}$ & $c_{4}$ & $c_{5}$ & $c_{6}$ & $c_{7}$ & $c_{8}$ \\
\hline$w^{*}$ & 0.134 & 0.100 & 0.067 & 0.330 & 0.080 & 0.201 & 0.028 & 0.057 \\
Rank & 3 & 4 & 6 & 1 & 5 & 2 & 8 & 7 \\
$\xi^{R *}$ & 0.071 & & & & & & & \\
\hline
\end{tabular}

We used Eq. (2) to calculate the over scores of alternatives, the results are shown in Table 11.

Table 11

Overall score and ranking of each Alternative in the nominal problem of example 4

\begin{tabular}{llllllll}
\hline Alternative & Supplier A & Supplier B & Supplier C & Supplier D & Supplier E & Supplier F & Supplier G \\
\hline Overall score & 0.462 & 0.417 & 0.340 & 0.357 & 0.471 & 0.408 & 0.393 \\
\hline Rank & 2 & 3 & 7 & 6 & 1 & 4 & 5 \\
\hline
\end{tabular}

We use these results to compare the effect of uncertainty on our decisions. Note that since we have used only one respondent instead of six in the main model. Next, we study the effect of applying different and the same errors to pairwise comparisons in separate parts.

Part 1. In this part different random values of error are added to each comparison. here we trust the respondent but we assume that for each criterion, his/her opinion contains some errors. The main purpose is to make our decision robust against the varying parameters. The value of each error is shown in Table 12. We must apply these errors to the pairwise comparisons (Table 8).

Table 12

The value of errors $(e)$ for part 1 in example 4

\begin{tabular}{|c|c|c|c|c|c|c|c|c|}
\hline $\mathrm{BO}$ & $c_{1}$ & $c_{2}$ & $c_{3}$ & $C_{4}$ & $C_{5}$ & $c_{6}$ & $c_{7}$ & $C_{8}$ \\
\hline Error & \pm 0.11 & \pm 0.40 & \pm 0.02 & \pm 0 & \pm 1 & \pm 0.005 & \pm 0.33 & \pm 0.91 \\
\hline $\mathrm{OW}$ & & & & & & & & Error \\
\hline$c_{1}$ & & & & & & & & \pm 0.95 \\
\hline$c_{2}$ & & & & & & & & \pm 0.96 \\
\hline$c_{3}$ & & & & & & & & \pm 0.15 \\
\hline$c_{4}$ & & & & & & & & \pm 0.97 \\
\hline$c_{5}$ & & & & & & & & \pm 0.09 \\
\hline$c_{6}$ & & & & & & & & \pm 0.48 \\
\hline$c_{7}$ & & & & & & & & \pm 0 \\
\hline$c_{8}$ & & & & & & & & \pm 0.16 \\
\hline
\end{tabular}

Solving the problem (10) with $(e \neq 0, \Omega=5.24)$ yields the optimal weights and the consistency indicator. The results are shown in Table13.

Table 13

Weights and rankings of each criterion for the part 1 of example 4

\begin{tabular}{lllllllll} 
Criterion & $c_{1}$ & $c_{2}$ & $c_{3}$ & $c_{4}$ & $c_{5}$ & $c_{6}$ & $c_{7}$ & $c_{8}$ \\
\hline$w^{*}$ & 0.137 & 0.097 & 0.071 & 0.327 & 0.071 & 0.213 & 0.028 & 0.054 \\
Rank & 3 & 4 & 5 & 1 & 5 & 2 & 8 & 7 \\
$\xi^{R *}$ & 0.100 & & & & & & & \\
\hline
\end{tabular}

These results show that applying uncertainty changed the ranking of the criteria (for $c_{3}$ ). We lost less than $3 \%$ of the consistency because of considering errors in the parameters. We want to study the effect 
of uncertainty on the ranking of the alternatives, hence we must calculate the overall score of each alternative. Using Eq. (2), the results are shown in Table 14 and Fig. 3.

Table 14

Overall score and ranking of each alternative for part 1 of example 4

\begin{tabular}{llllllll}
\hline Alternative & Supplier A & Supplier B & Supplier C & Supplier D & Supplier E & Supplier F & Supplier G \\
\hline Overall score & 0.473 & 0.421 & 0.345 & 0.354 & 0.472 & 0.408 & 0.390 \\
Rank & 1 & 3 & 7 & 6 & 2 & 4 & 5 \\
\hline
\end{tabular}

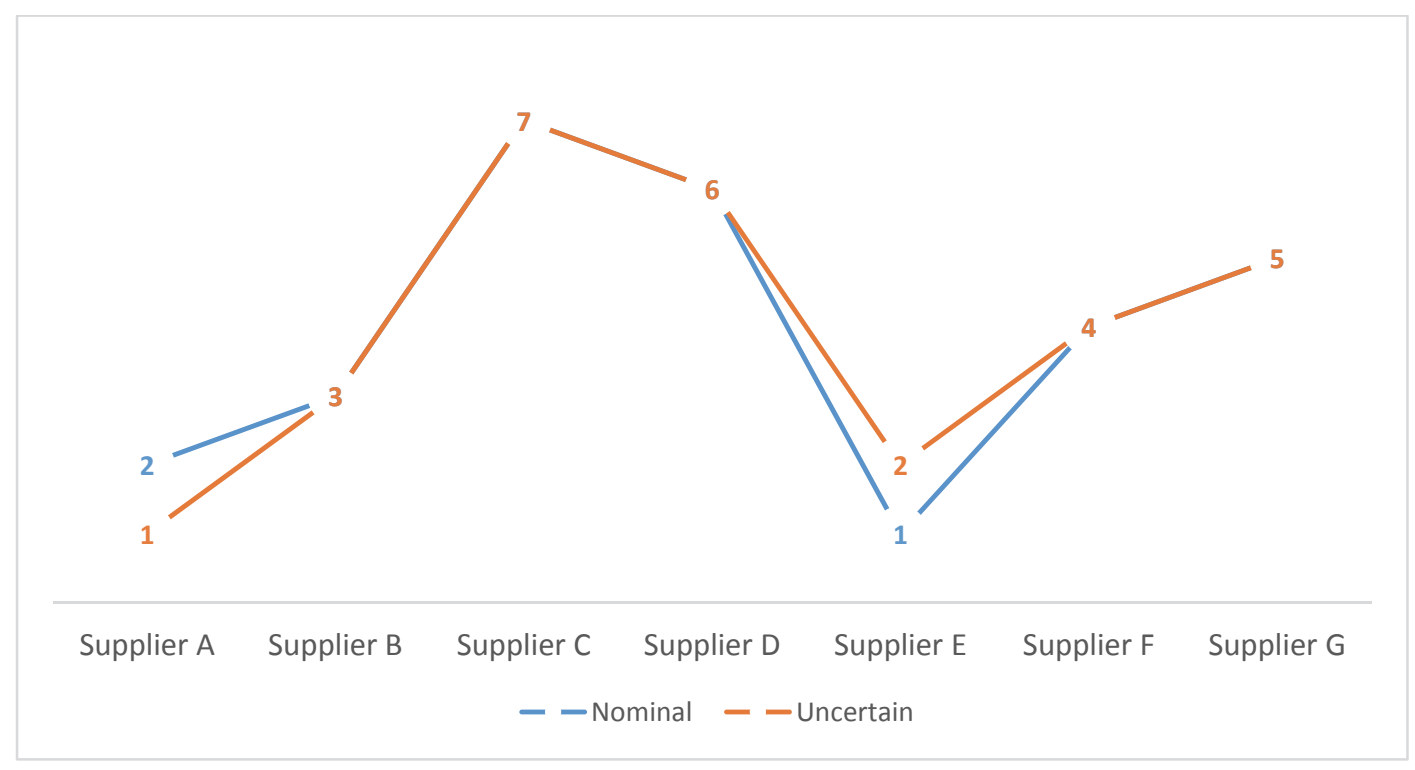

Fig. 3. The rankings of the alternatives in the nominal and uncertain problem of part 1

Comparing Table 14 with Table 11, an interesting finding is obtained. Assume that we want to choose one supplier. If we study the nominal problem, the supplier E will be chosen. Applying uncertainty to the problem yields completely different results, since under uncertain problem we will choose supplier A as the best supplier with the highest overall score. This shows when we trust on respondent's opinion but we think he/she may have some errors while assigning values to each comparison, it is necessary to consider uncertainty to make a rational decision.

Part 2. In this part, we want to add the same errors to all pairwise comparisons. We consider that there is a certain error in the opinion of the respondent and we cannot have full trust on him/her (it may occur when historical data show that the respondent had a certain error on previous works). However, we must add the same error to each pairwise comparison.

Assume that according to the historical data we can trust the respondent for $80 \%$ of the time, so we must add $20 \%$ error to all pairwise comparisons. (in a scale of 1 to $9,20 \%$ is 1.6 hence the error is \pm 1.6 for all values in the vectors $\mathrm{BO}$ and $\mathrm{OW}$ )

We have solved the problem (10), with $e \neq 0, \Omega=5.24$, the optimal weights, consistency indicator and the ranking of the criteria are shown in Table 15.

Table 15

Weight and ranking of each criterion for the part 2 of example 4.

\begin{tabular}{lllllllll}
\hline Criterion & $c_{1}$ & $c_{2}$ & $c_{3}$ & $c_{4}$ & $c_{5}$ & $c_{6}$ & $c_{7}$ & $c_{8}$ \\
\hline$w^{*}$ & 0.127 & 0.104 & 0.077 & 0.326 & 0.088 & 0.163 & 0.044 & 0.068 \\
Rank & 3 & 4 & 6 & 1 & 5 & 2 & 8 & 7 \\
$\xi^{R *}$ & 0.260 & & & & & & & \\
\hline
\end{tabular}


When we compare these results with the nominal results, we find that the ranking of the criteria did not change, but we must investigate the ranking of the alternatives. Note that the inconsistency indicator increased three times but, we have about $74 \%$ consistency and our results are reliable.

Using Eq. (2) yields the results which are shown in Table 16 and Fig. 4.

Table 16

Overall score and ranking of each alternative for part 2 of example 4

\begin{tabular}{llllllll}
\hline Alternative & Supplier A & Supplier B & Supplier C & Supplier D & Supplier E & Supplier F & Supplier G \\
\hline Overall score & 0.453 & 0.410 & 0.343 & 0.370 & 0.476 & 0.414 & 0.399 \\
Rank & 2 & 4 & 7 & 6 & 1 & 3 & 5 \\
\hline
\end{tabular}

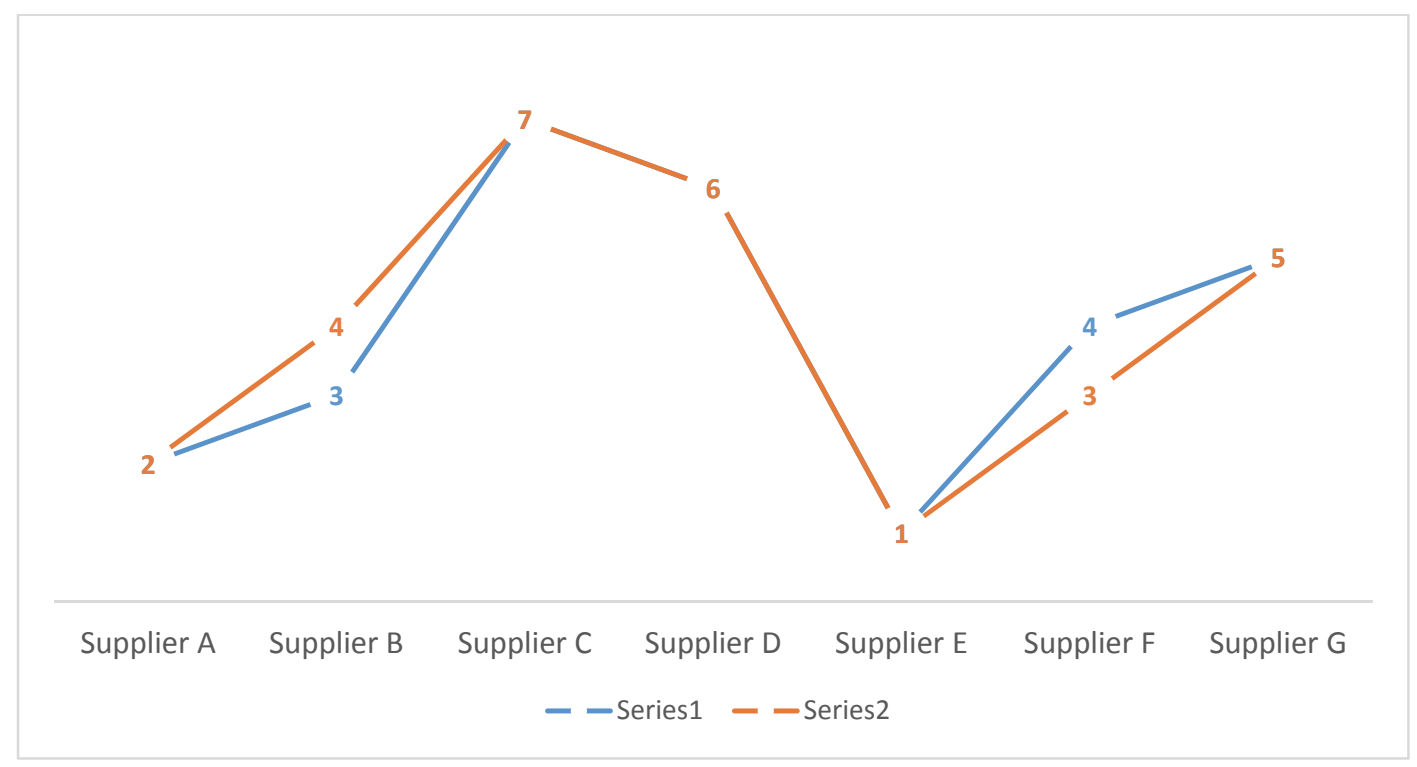

Fig. 4. The rankings of the alternatives in the nominal and the uncertain problem of part 2

According to the results we have obtained different rankings for two suppliers (F, B) and it shows that when we cannot fully trust in a respondent (even when we can trust him/her in $80 \%$ of the time), we must apply uncertainty to our decision for a better response to an MCDM problem.

The results show that applying uncertainty would change the ranking of the alternatives, but if we want to determine how much uncertainty would make changes in the results, we must find the point which will cause the changes. We solved the problem (10) with $e=16.3 \%$, the results are shown in Table 17 and Fig. 5. Note that $e=16.3 \%$ is the first point which the changes will occur.

\section{Table 17}

Overall score and ranking of each alternative for $e=16.3 \%$ and $\Omega=5.24$ in part 2 of example 4

\begin{tabular}{llllllll}
\hline Alternative & Supplier A & Supplier B & Supplier C & Supplier D & Supplier E & Supplier F & Supplier G \\
\hline Overall score & 0.452 & 0.4098 & 0.340 & 0.365 & 0.471 & 0.4098 & 0.395 \\
Rank & 2 & 3 & 7 & 6 & 1 & 3 & 5 \\
\hline
\end{tabular}

By comparing this results with Table 11, we find that in this example when our trust on the respondent is not over $83.7 \%$, applying uncertainty would change the ranking of alternatives (supplier F ranked $3 \mathrm{rd}$ in the uncertain problem and it ranked 4th in the nominal problem). This result shows the importance of paying attention to the uncertainty in decision-making problems.

As we argued so far, experimental results show that applying uncertainty to the MCDM problems may cause major changes in the ranking of the criteria and alternatives. Since the data used in the MCDM 
problems is usually qualitative (for example pairwise comparisons) it is rational to study these problems as an uncertain problem and make our decisions robust against the error of unreliable data.

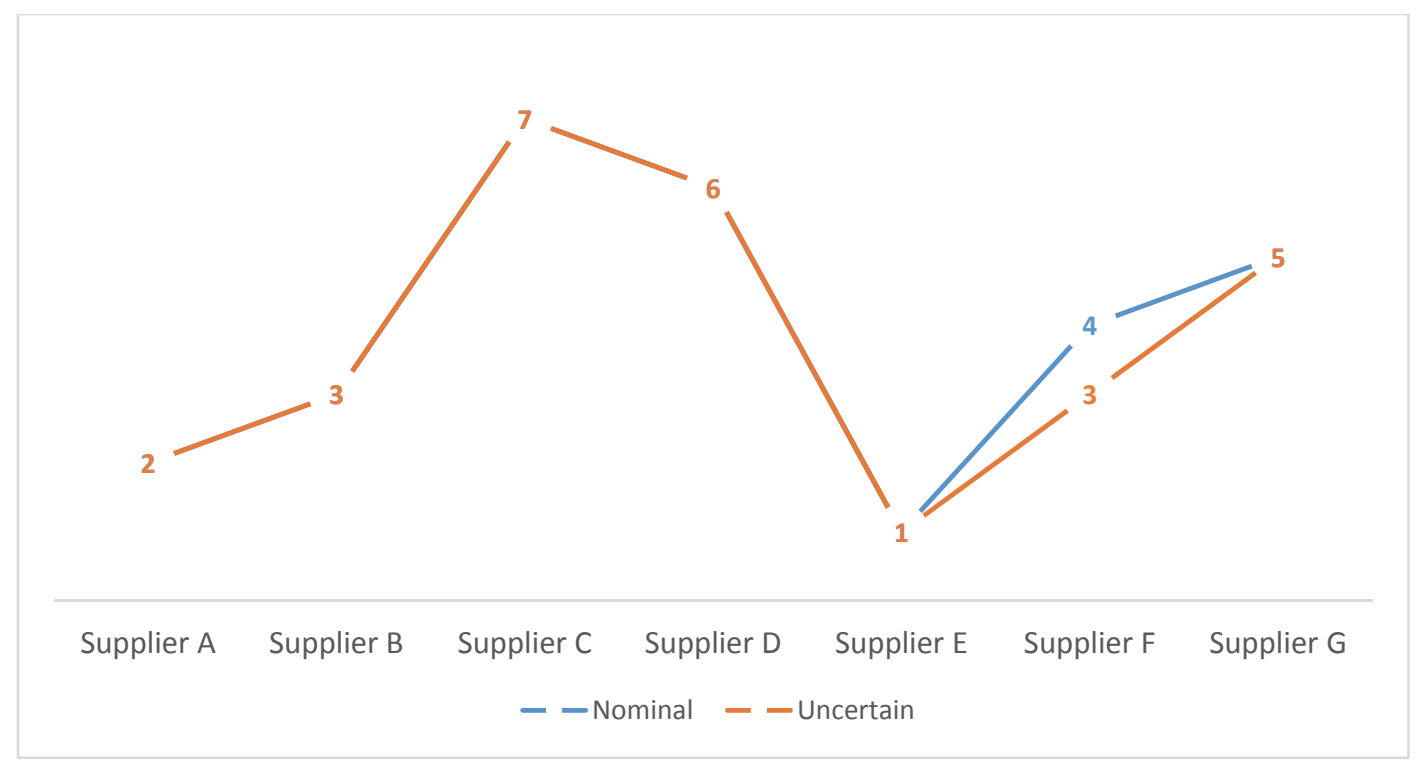

Fig. 5. The rankings of the alternatives in the nominal and the uncertain problem for $e=16.3 \%$

\section{Conclusion}

In this paper, we have proposed a robust approach for MCDM problems. The presented method was based on the Best-Worst Method (BWM) which uses pairwise comparisons to derive the optimal weights of different criteria. We have presented different robust approaches for BWM when the pairwise comparisons data were not reliable and contained some errors. Then we developed a linear programming of Ben-Tal's robust formulation for BWM. We have shown that it is better to use BenTal's method because we have more control over the uncertainty than Soyester's method and we can obtain more reliable results than Bertsimas' approach. These methods can be used when the decisionmaker cannot assign an exact value to each comparison and wants to assign intervals to the comparisons. The results are the same as the nominal problem results for zero protection (error $=0$ ) and this shows the performance of the uncertain methods. The experimental results show that applying uncertainty will change the order of criteria and alternatives. So, for a rational decision-making we must add uncertainty to the nominal problem. We have found that whether the same or different, adding error to each comparison may change the ranking of the alternatives and affect our decision. Although adding same errors to comparisons will not change the ranking of the criteria, it can change the ranking of the alternatives. According to the results, even a minor error in data will change the decisions and this shows the importance of studying the uncertainty in this field. As a general result of this paper, we have found that applying uncertainty to MCDM problem is necessary. We suggest extending this method for group decision-making problems. Integrating this method with other methods such as scenario-based methods can be a future research. As a future work in this field, we can compare these methods with other ones and apply the methods to some real cases to show the validation of the proposed models.

\section{Supplementary data}

This paper is supported with detailed working codes written in MATLAB and they can be downloaded from http://www.growingscience.com/ds1/Vol7/sup.zip 


\section{Acknowledgement}

The authors would like to thank the reviewers for their constructive comments on earlier version of this paper.

\section{References}

Abadi, F., Sahebi, I., Arab, A., Alavi, A., \& Karachi, H. (2018). Application of best-worst method in evaluation of medical tourism development strategy. Decision Science Letters, 7(1), 77-86.

Ahmad, W. N. K. W., Rezaei, J., Sadaghiani, S., \& Tavasszy, L. A. (2017). Evaluation of the external forces affecting the sustainability of oil and gas supply chain using Best Worst Method. Journal of Cleaner Production, 153, 242-252.

Askarifar, K., Motaffef, Z., \& Aazaami, S. (2018). An investment development framework in Iran's seashores using TOPSIS and best-worst multi-criteria decision making methods. Decision Science Letters, 7(1), 55-64.

Ben-Tal, A., \& Nemirovski, A. (2000). Robust solutions of linear programming problems contaminated with uncertain data. Mathematical programming, 88(3), 411-424.

Bertsimas, D., \& Sim, M. (2004). The price of robustness. Operations research, 52(1), 35-53.

Ghaffari, S., Arab, A., Nafari, J., \& Manteghi, M. (2017). Investigation and evaluation of key success factors in technological innovation development based on BWM. Decision Science Letters, 6(3), 295-306.

Guo, S., \& Zhao, H. (2017). Fuzzy best-worst multi-criteria decision-making method and its applications. Knowledge-Based Systems, 121, 23-31.

Gupta, H. (2017). Evaluating service quality of airline industry using hybrid best worst method and VIKOR. Journal of Air Transport Management. https://doi.org/10.1016/j.jairtraman.2017.06.001

Gupta, P., Anand, S., \& Gupta, H. (2017). Developing a roadmap to overcome barriers to energy efficiency in buildings using best worst method. Sustainable Cities and Society, 31, 244-259.

Gupta, H., \& Barua, M. K. (2016). Identifying enablers of technological innovation for Indian MSMEs using best-worst multi criteria decision making method. Technological Forecasting and Social Change, 107, 69-79.

Gupta, H., \& Barua, M. K. (2017). Supplier selection among SMEs on the basis of their green innovation ability using BWM and fuzzy TOPSIS. Journal of Cleaner Production, 152, 242-258.

Hafezalkotob, A., \& Hafezalkotob, A. (2017). A novel approach for combination of individual and group decisions based on fuzzy best-worst method. Applied Soft Computing, 59, 316-325.

Hwang, C. L., \& Yoon, K. (1981). Methods for multiple attribute decision making. In Multiple attribute decision making (pp. 58-191). Springer, Berlin, Heidelberg.

Mohaghar, A., Sahebi, I. G., \& Arab, A. (2017). Appraisal of humanitarian supply chain risks using best-worst method. International Journal of Social, Behavioral, Educational, Economic, Business and Industrial Engineering, 11(2), 309-314.

Mou, Q., Xu, Z., \& Liao, H. (2016). An intuitionistic fuzzy multiplicative best-worst method for multicriteria group decision making. Information Sciences, 374, 224-239.

Opricovic, S., \& Tzeng, G. H. (2007). Extended VIKOR method in comparison with outranking methods. European Journal of Operational Research, 178(2), 514-529.

Ren, J., Liang, H., \& Chan, F. T. (2017). Urban sewage sludge, sustainability, and transition for EcoCity: Multi-criteria sustainability assessment of technologies based on best-worst method. Technological Forecasting and Social Change, 116, 29-39.

Rezaei, J. (2015). Best-worst multi-criteria decision-making method. Omega, 53, 49-57.

Rezaei, J., Wang, J., \& Tavasszy, L. (2015). Linking supplier development to supplier segmentation using Best Worst Method. Expert Systems with Applications, 42(23), 9152-9164.

Rezaei, J. (2016). Best-worst multi-criteria decision-making method: Some properties and a linear model. Omega, 64, 126-130. 
Rezaei, J., Nispeling, T., Sarkis, J., \& Tavasszy, L. (2016). A supplier selection life cycle approach integrating traditional and environmental criteria using the best worst method. Journal of Cleaner Production, 135, 577-588.

Roy, B. (1968). Classement et choix en présence de points de vue multiples. Revue franiaise d'informatique et de recherche opérationnelle, 2(8), 57-75.

Salimi, N., \& Rezaei, J. (2016). Measuring efficiency of university-industry Ph. D. projects using best worst method. Scientometrics, 109(3), 1911-1938.

Salimi, N. (2017). Quality assessment of scientific outputs using the BWM. Scientometrics, 112(1), 195-213.

Salimi, N., \& Rezaei, J. (2018). Evaluating firms' R\&D performance using best worst method. Evaluation and Program Planning, 66, 147-155.

Shojaei, P., Haeri, S. A. S., \& Mohammadi, S. (2017). Airports evaluation and ranking model using Taguchi loss function, best-worst method and VIKOR technique. Journal of Air Transport Management.

Soyster, A. L. (1973). Convex programming with set-inclusive constraints and applications to inexact linear programming. Operations Research, 21(5), 1154-1157.

van de Kaa, G., Kamp, L., \& Rezaei, J. (2017). Selection of biomass thermochemical conversion technology in the Netherlands: A best worst method approach. Journal of Cleaner Production, 166, 32-39.

van de Kaa, G., Scholten, D., Rezaei, J., \& Milchram, C. (2017). The Battle between Battery and Fuel Cell Powered Electric Vehicles: A BWM Approach. Energies, 10(11), 1707.

Yang, Q., Zhang, Z., You, X., \& Chen, T. (2016). Evaluation and classification of overseas talents in China based on the BWM for intuitionistic relations. Symmetry, 8(11), 137.

You, X., Chen, T., \& Yang, Q. (2016). Approach to multi-criteria group decision-making problems based on the best-worst-method and electre method. Symmetry, 8(9), 95

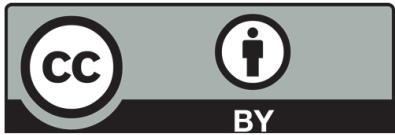

(C) 2018 by the authors; licensee Growing Science, Canada. This is an open access article distributed under the terms and conditions of the Creative Commons Attribution (CC-BY) license (http://creativecommons.org/licenses/by/4.0/). 